\title{
Neutrophil-to-lymphocyte ratio independently predicts advanced pathological staging and poorer survival outcomes in testicular cancer
}

\author{
Yu Guang Tan, Joshua Sia, Hong Hong Huang, Weber Kam On Lau \\ Department of Urology, Singapore General Hospital, Singapore
}

Purpose: An elevated neutrophil-to-lymphocyte ratio (NLR) has been associated with adverse outcomes in various malignancies. However, its role in prognosticating testicular cancer (TC) has not been validated. We aim to study the relationship between NLR and TC.

Materials and Methods: We retrospectively reviewed 160 patients with histological proven TC from January 2005 to June 2016. Youden's index was used to analyse NLR and a cut-off point of 3.0 was obtained, with statistical receiver operating characteristics of 0.755 . Chi-square test, Kaplan-Meier (log rank test) and logistics regression models were used to predict NLR association with survival outcomes.

Results: Median age was 34 years old (range, 17-68 years old). There were 102 pure seminomas and 58 non-seminomatous germ cell tumours. Median follow-up period was 8 years (range, $2.5-17$ years). NLR $\geq 3.0$ was independently associated with lymph node involvement $(\mathrm{p}=0.031$; odds ratio [OR], 2.91; 95\% confidence interval $[\mathrm{Cl}], 1.67-5.83 ; \mathrm{p}=0.038 ; \mathrm{OR}, 4.12 ; 95 \% \mathrm{Cl}, 1.26-6.51)$ and metastatic disease $(\mathrm{p}=0.041 ; \mathrm{OR}, 2.48 ; 95 \% \mathrm{Cl}, 1.22-3.98 ; \mathrm{p}=0.043 ; \mathrm{OR}, 2.21 ; 95 \% \mathrm{Cl}, 1.17-3.65)$ in both seminomatous and nonseminomatous germ cell tumours, translating to a more advanced disease. Moreover, NLR $\geq 3.0$ also predicts poorer cancer specific survival in these patients.

Conclusions: NLR can be an inexpensive haematological marker in predicting advanced TC staging and poorer survival outcome. NLR complements the traditional cancer staging by identifying a group of high risk patients who may benefit from multimodal treatment and closer surveillance to achieve long term survival.

Keywords: Lymphocytes; Neutrophils; Testicular neoplasms

This is an Open Access article distributed under the terms of the Creative Commons Attribution Non-Commercial License (http://creativecommons.org/licenses/by-nc/4.0) which permits unrestricted non-commercial use, distribution, and reproduction in any medium, provided the original work is properly cited.

\section{INTRODUCTION}

Testicular cancer (TC) is a relatively rare malignancy, with an estimated annual incidence of 5.7 per 100,000 men [1,2], representing $1 \%$, or 24 th commonest, of all male malignant conditions. However, TC is the most common cancer form in men between aged 15 to 44 years in many countries that have attained high scores on the human development index, reflecting important psychosocial and economic problems in these otherwise healthy age groups

Received: 7 January, 2019 - Accepted: 1 March, 2019

Corresponding Author: Yu Guang Tan

Department of Urology, Singapore General Hospital, 12 Woodgrove Drive \#01-48, Singapore 738212

TEL: +65-97392285, FAX: +65-63266808, E-mail: yuguangtan90@gmail.com

ORCID: https://orcid.org/0000-0002-8166-0582 
[3]. Survival outcomes are favourable for TC, with $95 \%$ of patients achieving 5 -year cancer specific survival (CSS). This is attributed to the predominantly early clinical staging at diagnosis of TC. Only 10\% of TC presents with metastatic disease, and in these patients, 5-year CSS drops to 73\% [4].

Interestingly, cumulative evidence has supported the role of inflammation in cancer development and progression [5], and the neutrophil-to-lymphocyte ratio (NLR), represents an inexpensive, easily measured and reproducible marker of systemic inflammation that correlates with tumour activity [6]. A higher NLR has been previously demonstrated to be associated with poorer disease-specific and overall survival in gastric, hepatic, non-small cell and cervical cancer [7-9]. More recent studies have reproduced similar associations in urological malignancies [10-13].

However, limited data exist regarding the potential prognostic role of NLR in TC [14,15], Moreover, these studies are limited by the small numbers, short follow-up and mixed outcomes. As such, in this paper, we sought to determine the association between preoperative NLR and pathologic outcomes and survival outcomes. In particularly, in patients with metastatic TC, we hope that NLR can identify a particular subgroup that may harbour a more aggressive tumour biology. These patients may subsequently benefit from closer monitoring and adjuvant therapies.

\section{MATERIALS AND METHODS}

Following Singhealth Institutional Review Board approval, we retrospectively reviewed our institution's cancer registry database and identified 160 patients diagnosed with TC from January 2005 to June 2016 (approval number: 2009/1053/D). Written informed consent was waived by the board. All patients underwent an initial ultrasound scrotum which raised the suspicion of an underlying testicular neoplasm. From there, they all received 1) standard inguinal radical orchiectomy; 2) computed tomography scan of the thorax, abdomen and pelvis; 3) serum tumour markers (alpha fetoprotein, lactate dehydrogenase and beta-human chorionic gonadotropin) as part of the complete staging workup. The $\mathrm{T}$ staging was determined on final histology after the standard inguinal radical orchidectomy, whereas the $\mathrm{N}$ and $\mathrm{M}$ stagings were determined clinically on imaging modalities.

Hematological and biochemical blood results were collected at a median of 6 days prior to radical orchidectomy, as part of our routine pre-operative investigations. Tumour markers were collected at a median of 18 days after radical orchidectomy to complete the staging. No patient received chemotherapy prior to these blood results. Twelve patients with active inflammatory and infective conditions that could affect the NLR were excluded. Of which, 4 patients had concomitant systemic lupus erythematosus, 4 had active rheumatoid arthritis, 2 had ankylosing spondylitis and 2 had Sjogren's syndrome.

The treatment plan for most patients was standardized. All patients who had stage I disease were put on closer surveillance with no adjuvant therapy. Patients with stage II and III diseases (59 patients) were routinely referred to our medical oncologists. These patients were risk stratified according to the International Germ Cell Cancer Collaborative Group and underwent the necessary adjuvant chemotherapies. Specifically, patients risk stratified as 'good prognosis' underwent 3 cycles of bleomycin, etoposide and cisplatin (BEP), and those stratified as 'intermediate to poor prognosis' underwent 4 cycles of BEP. No patient received radiotherapy as primary adjuvant modality over chemotherapy. Altogether, 48 patients achieved complete remission while 11 patients achieved partial remission upon completion of chemotherapy. Given the retrospective nature of this study, coupled with the heterogeneity of physicians' practices, patients' preferences and cost issues, subsequent follow-up plans were not consistently standardized. Our institution recommended a 3 monthly outpatient visit for the first 2 years, 6 monthly for the 3rd year, and subsequent yearly visit. Serum tumour markers were repeated upon each visit and computed tomography scan was scheduled yearly for the first 5-years. Over a median follow-up time was 8 years (range, 2.5-17 years), 10 patients (6.3\%) developed recurrence, of which $8(5.0 \%)$ had died of metastatic disease. We defined recurrence as the development of new lymph node involvement (LNI) or metastatic sites after a period of surveillance in stage I disease, and after a period of disease remission in stage $I I$ and $\mathrm{II}$ diseases.

The primary outcome was to evaluate the correlation between NLR and clinicopathological outcomes, predominantly the extent of disease burden measured by LNI and metastasis. Because LNI and metastasis are confirmed via imaging modalities and subsequently treated with adjuvant chemotherapy, these variables were analysed as clinical outcomes. Secondary outcome was the CSS.

Statistical analyses were performed using IBM SPSS Statistics ver. 20.0 (IBM Co., Armonk, NY, USA). Youden's index was used to determine the relationship between NLR and extra-testicular disease, defined as either nodal of metastatic involvement, which translate to stage II and above. A cut-off point of 3.0 was obtained, with a statistical receiver operating characteristics (ROCs) of 0.755 (area under 
curve) (Fig. 1). The sensitivity at this cut-off point was $74.4 \%$, specificity of $77.8 \%$, positive predictive value of $56.2 \%$ and negative predictive value of $89.2 \%$. The relationship between clinicopathological features and NLR was calculated with chi-square test. Multivariate analyses were performed using logistics regression models. Survival curves were calculated with the Kaplan-Meier log rank analysis. All tests were two-sided with $\mathrm{p}<0.05$ considered to be statistically significant.

\section{RESULTS}

The patients' demographics and tumour characteristics were shown in Table 1. Upon completion of the staging workup, the pathological T staging was as followed: 109 patients (68.1\%) had T1, 40 patients (25.0\%) had T2 and 11 patients (6.9\%) had T3 disease. The median tumour size was $5.7 \mathrm{~cm}$ (range, 1.2-11.8 cm), of which 117 patients (73.1\%) had tumour size $>4 \mathrm{~cm}$. One hundred and two patients $(63.8 \%)$ had pure seminomas while the other 58 patients (36.3\%) had non-seminomatous germ cell tumours (NSGCTs). Fifty-five patients (34.4\%) had clinical LNI while 23 patients (14.4\%) had clinical visceral or bone metastasis. In accordance to the American Joint Committee on Cancer (AJCC) staging (8th edition) of TC, 101 patients (63.1\%) had stage I disease, while 27 (16.9\%) had stage II and 32 patients (20.0\%) had stage III disease.

\section{NLR and pathological outcomes}

The clinicopathologic features of patients with NLR $<3.0$ and $\geq 3.0$ were shown in Table 2 . In both seminomas and NSGCTs, patients with NLR $\geq 3.0$ were associated with increased likelihood of LNI, metastatic disease and hence, an overall higher cancer staging. These associations remained

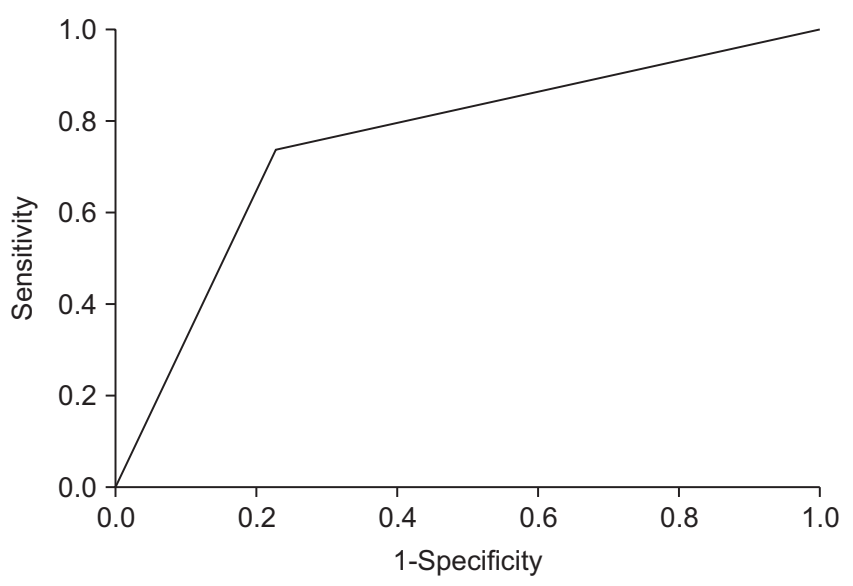

Fig. 1. Receiver operating characteristic (ROC) curve. significant on multivariate analyses. For instance, NLR $\geq 3.0$ was associated with $3 \mathrm{x}$ ( $\mathrm{p}=0.031$; odds ratio [OR], 2.91; 95\% confidence interval [CI], 1.67-5.83) and 4x ( $\mathrm{p}=0.038$; OR, 4.12; 95\% CI, 1.26-6.51) the likelihood of LNI in patients with pure seminomas or NSGCTs respectively (Table 3). Similarly, $\mathrm{NLR} \geq 3.0$ was associated with $2.5 \mathrm{x}$ ( $\mathrm{p}=0.041$; OR, 2.48; 95\%

Table 1. Patient demographics and tumour characteristics $(n=160)$

\begin{tabular}{|c|c|}
\hline Variable & Value \\
\hline Age $(y)$ & $34(17-68)$ \\
\hline Body mass index $\left(\mathrm{kg} / \mathrm{m}^{2}\right)$ & $23.3(15.7-31.5)$ \\
\hline \multicolumn{2}{|l|}{ Tumour size $(\mathrm{cm})$} \\
\hline$\leq 4$ & $43(26.9)$ \\
\hline$>4$ & $117(73.1)$ \\
\hline \multicolumn{2}{|l|}{ Histology } \\
\hline Seminoma & $102(63.8)$ \\
\hline NSGCT & $58(36.3)$ \\
\hline \multicolumn{2}{|l|}{ Pathological tumor staging } \\
\hline $\mathrm{T} 1$ & $109(68.1)$ \\
\hline $\mathrm{T} 2$ & $40(25.0)$ \\
\hline T3 & $11(6.9)$ \\
\hline \multicolumn{2}{|l|}{ Nodal disease } \\
\hline No & $105(65.6)$ \\
\hline $\mathrm{N}+$ & $55(34.4)$ \\
\hline \multicolumn{2}{|l|}{ Metastasis } \\
\hline Mo & $137(85.6)$ \\
\hline $\mathrm{M}+$ & $23(14.4)$ \\
\hline \multicolumn{2}{|l|}{ Serum tumour markers } \\
\hline So & $58(36.3)$ \\
\hline S1 & $57(35.6)$ \\
\hline S2 & $36(22.5)$ \\
\hline S3 & $9(5.6)$ \\
\hline \multicolumn{2}{|l|}{ AJCC staging } \\
\hline Stage I & $101(63.1)$ \\
\hline Stage II & $27(16.9)$ \\
\hline Stage III & $32(20.0)$ \\
\hline Neutrophils & $63.8(29.7-88.9)$ \\
\hline Lymphocytes & $25.3(4.8-49.8)$ \\
\hline NLR & $2.68(0.7-18.2)$ \\
\hline \multicolumn{2}{|l|}{ NLR breakdown } \\
\hline$<3.0$ & $102(63.8)$ \\
\hline$\geq 3.0$ & $58(36.3)$ \\
\hline Follow-up time $(y)$ & $8(2.5-17)$ \\
\hline \multicolumn{2}{|l|}{ Recurrence } \\
\hline Yes & $10(6.3)$ \\
\hline No & $150(93.8)$ \\
\hline \multicolumn{2}{|l|}{ Disease status } \\
\hline Alive & $152(95.0)$ \\
\hline Dead & $8(5.0)$ \\
\hline
\end{tabular}

Values are presented as median (range) or number (\%). NSGCT, non-seminomatous germ cell tumour; AJCC, American Joint Committee on Cancer; NLR, neutrophil-to-lymphocyte ratio. 
Table 2. Clinicopathologic characteristics and pathological outcomes for seminomas and NSGCTs

\begin{tabular}{|c|c|c|c|c|c|c|c|c|}
\hline & \multicolumn{4}{|c|}{ Seminomas } & \multicolumn{4}{|c|}{ NSGCT } \\
\hline & NLR $<3.0$ & $N L R \geq 3.0$ & p-value & $95 \% \mathrm{Cl}$ & NLR $<3.0$ & $N L R \geq 3.0$ & $p$-value & $95 \% \mathrm{Cl}$ \\
\hline Size $(\mathrm{cm})$ & & & 0.594 & & & & 0.970 & \\
\hline$\leq 4$ & 14 & 5 & & & 14 & 10 & & \\
\hline$>4$ & 54 & 29 & & & 20 & 14 & & \\
\hline \multicolumn{9}{|l|}{ T staging } \\
\hline $\mathrm{T} 1$ & 45 & 26 & & & 28 & 10 & & \\
\hline $\mathrm{T} 2$ & 21 & 6 & 0.179 & & 5 & 8 & 0.072 & \\
\hline T3 & 2 & 2 & 0.483 & & 1 & 6 & 0.013 & $6.28(2.05-9.51)$ \\
\hline Nodal disease & & & 0.005 & $3.76(1.53-9.26)$ & & & $<0.01$ & $11.70(4.03-16.63)$ \\
\hline NO & 55 & 18 & & & 27 & 5 & & \\
\hline $\mathrm{N}+$ & 13 & 16 & & & 7 & 19 & & \\
\hline Metastatic disease & & & 0.041 & $6.83(1.42-11.53)$ & & & $<0.01$ & $13.01(6.07-19.57)$ \\
\hline Mo & 67 & 30 & & & 32 & 8 & & \\
\hline M1 & 1 & 4 & & & 2 & 16 & & \\
\hline S staging & & & 0.423 & & & & $<0.01$ & 6.75 (2.11-9.86) \\
\hline $0-1$ & 57 & 26 & & & 25 & 7 & & \\
\hline $2-3$ & 11 & 8 & & & 9 & 17 & & \\
\hline \multicolumn{9}{|l|}{ Staging } \\
\hline Stage 1 & 54 & 18 & & & 26 & 3 & & \\
\hline Stage 2A/B (low volume metastasis) & 10 & 10 & 0.036 & $3.00(1.12-8.40)$ & 3 & 3 & 0.034 & $8.69(2.41-13.84)$ \\
\hline Stage 2C/3 (advanced metastasis) & 4 & 6 & 0.032 & $4.50(1.18-12.86)$ & 5 & 18 & $<0.01$ & $13.60(6.62-19.58)$ \\
\hline Recurrence & & & 0.112 & & & & 0.220 & \\
\hline Yes & 1 & 3 & & & 2 & 4 & & \\
\hline No & 67 & 31 & & & 32 & 20 & & \\
\hline
\end{tabular}

NSGCT, non-seminomatous germ cell tumour; NLR, neutrophil-to-lymphocyte ratio; Cl, confidence interval.

Table 3. Factors associated with lymph node involvement

\begin{tabular}{|c|c|c|c|c|c|c|}
\hline & \multicolumn{3}{|c|}{ Seminomas } & \multicolumn{3}{|c|}{ NSGCT } \\
\hline & \multirow{2}{*}{$\begin{array}{c}\text { Univariate } \\
\mathrm{p} \text {-value }\end{array}$} & \multicolumn{2}{|c|}{ Multivariate } & \multirow{2}{*}{$\begin{array}{c}\text { Univariate } \\
\mathrm{p} \text {-value }\end{array}$} & \multicolumn{2}{|c|}{ Multivariate } \\
\hline & & p-value & OR $(95 \% \mathrm{Cl})$ & & p-value & OR $(95 \% \mathrm{CI})$ \\
\hline Size $>4 \mathrm{~cm}$ & 0.576 & 0.580 & & 0.426 & 0.893 & \\
\hline T staging & 0.058 & 0.230 & & $<0.01$ & 0.025 & $6.32(1.53-11.81)$ \\
\hline S staging & 0.404 & 0.687 & & $<0.01$ & 0.104 & \\
\hline$N L R \geq 3.0$ & $<0.01$ & 0.031 & $2.91(1.67-5.83)$ & $<0.01$ & 0.038 & $4.12(1.26-6.51)$ \\
\hline Metastatic disease & $<0.01$ & $<0.01$ & $8.95(6.12-15.87)$ & $<0.01$ & 0.018 & $10.24(7.83-16.55)$ \\
\hline
\end{tabular}

NSGCT, non-seminomatous germ cell tumour; OR, odds ratio; Cl, confidence interval; NLR, neutrophil-to-lymphocyte ratio.

CI, 1.22-3.98) and 2x ( $p=0.043 ;$ OR, 2.21; 95\% CI, 1.17-3.65) the likelihood of metastasis in these patients respectively (Table 4).

\section{NLR and survival outcomes}

The AJCC staging system (8th edition) showed a strong correlation with CSS in both seminomas and NSGCT. Patients with stage I and II disease had nearly 100\% 5-year CSS, but drops to $\sim 80 \%$ in stage III disease (Fig. 2). NLR $\geq 3.0$ was also independently associated with a poorer CSS in both types of germ cell tumours (Table 5), although statistical significant was not reached in the seminomatous group (Fig. 3). In the NSGCT group, the 5-year CSS was nearly 100\% in patients with NLR <3.0, but drops significantly to $\sim 76 \%$ in patients with $\mathrm{NLR} \geq 3.0$ ( $\mathrm{p}=0.037$; hazard ratio, 6.20 ; $95 \% \mathrm{CI}$, 1.834-13.16).

\section{DISCUSSION}

In a large cohort of patients with TC on long-term postoperative follow-up, we found that preoperative NLR was associated with advanced cancer staging, as well as 
Table 4. Factors associated with metastatic disease

\begin{tabular}{|c|c|c|c|c|c|c|}
\hline & \multicolumn{3}{|c|}{ Seminomas } & \multicolumn{3}{|c|}{ NSGCT } \\
\hline & \multirow{2}{*}{$\begin{array}{c}\text { Univariate } \\
\text { p-value }\end{array}$} & \multicolumn{2}{|c|}{ Multivariate } & \multirow{2}{*}{$\begin{array}{c}\text { Univariate } \\
\text { p-value }\end{array}$} & \multicolumn{2}{|c|}{ Multivariate } \\
\hline & & $p$-value & OR $(95 \% \mathrm{Cl})$ & & p-value & OR (95\% CI) \\
\hline Size $>4 \mathrm{~cm}$ & 0.936 & 0.831 & & 0.566 & 0.677 & \\
\hline T staging & 0.011 & 0.038 & $3.81(1.78-5.64)$ & $<0.01$ & 0.174 & \\
\hline S staging & 0.232 & 0.553 & & $<0.01$ & 0.150 & \\
\hline$N L R \geq 3.0$ & 0.024 & 0.041 & $2.48(1.22-3.98)$ & $<0.01$ & 0.043 & $2.21(1.17-3.65)$ \\
\hline Nodal disease & $<0.01$ & $<0.01$ & $11.18(8.42-18.46)$ & $<0.01$ & 0.028 & $9.68(5.32-14.58)$ \\
\hline
\end{tabular}

NSGCT, non-seminomatous germ cell tumour; OR, odds ratio; $\mathrm{Cl}$, confidence interval; NLR, neutrophil-to-lymphocyte ratio.

A

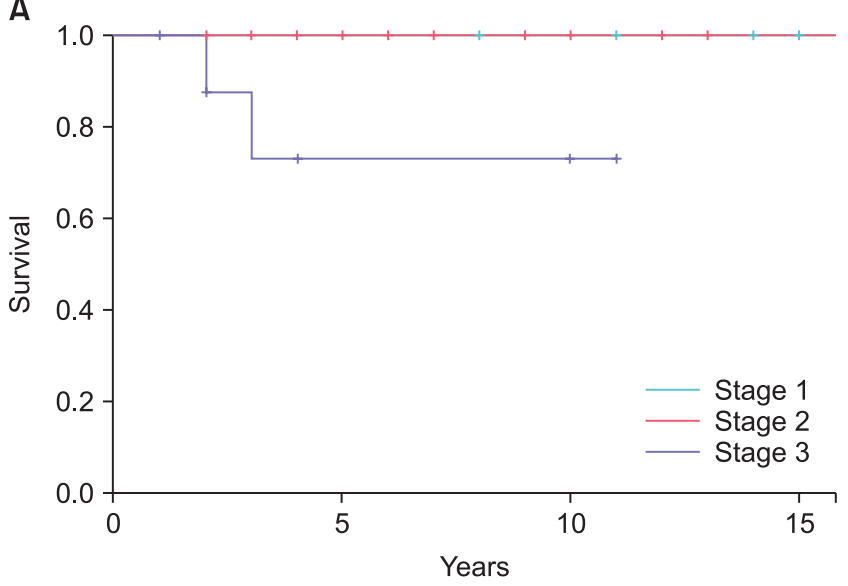

B

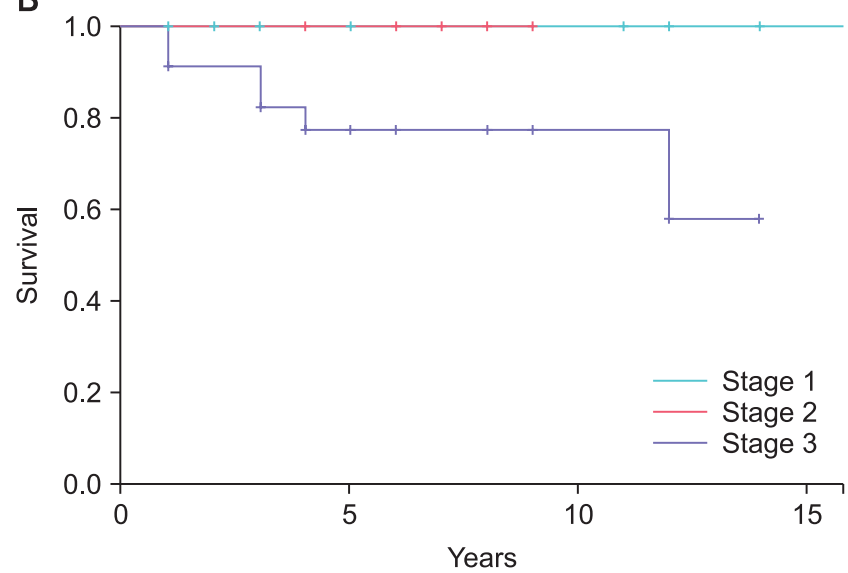

Fig. 2. (A) American Joint Committee on Cancer (AJCC) staging and cancer specific survival (CSS) in seminoma. (B) AJCC staging and CSS in non-seminomatous germ cell tumour (NSGCT).

poorer CSS. These findings remained significant after controlling for other clinicopathological features, suggesting an independent association of NLR with adverse outcomes.

The role of inflammation in tumour progression had been recognised in recent years [16]. Schepisi et al. [17] presented a very comprehensive discussion on the strong relationship between the role of immune system and inflammatory processes on the clinical outcome of patients with urothelial cancer. He detailed the pathophysiology between cytokines and NLR in tumour biology. A heightened circulating neutrophil level is associated with increased cytokines, particularly interleukins (IL-1, IL-6) and pro-angiogenic vascular endothelial growth factor which promote tumour migration and proliferation [18]. IL-6 protects the cancer cells from therapy-induced DNA damage, oxidative stress and apoptosis, hence promoting tumourigenesis [19]. On the other hand, a relative leukocytopenia, in particular cytotoxic $\mathrm{T}$ cells which are capable of targeted apoptotic cancer cell death, represents a weakened adaptive immune response to malignancy [20]. Hence, the combinatory effects of these two cellular mechanisms, or the NLR, may explain the tumour biology in cancers and predict survival outcomes.
Templeton et al. [21], in his meta-analysis of 100 studies, comprising 40,559 patients, demonstrated the strong relationship between NLR and over 20 solid tumours. Although the analysis involved a very heterogeneous group of malignancies, the sheer patient size and consistent results reflect the fundamental role of inflammation and immune suppression, as represented by NLR, in cancer biology. Since then, further independent studies, each focusing primarily on individual malignancy, or in particularly, urological malignancies, similarly established the significance of NLR. Ohno et al. [10] reviewed 198 patients who underwent radical nephrectomy and found a significant difference in 5-year recurrence free survival between NLR <2.7 (94\%) and $\geq 2.7$ (74\%) ( $\mathrm{p}=0.020)$. Jang et al. [11] evaluated 2,067 patients with prostate cancer undergoing radical prostatectomy and commented that a higher preoperative NLR $(\geq 1.76)$ predicted poorer CSS and overall survival (OS). Viers et al. [12] evaluated that in patients with urothelial bladder cancer undergoing radical cystectomy, higher preoperative NLR $(\geq 27)$ was associated with greater extravesical disease, LNI, higher recurrence rates and poorer CSS and OS.

Given the relative predictive value of NLR in other 
malignancies, its value in TC remains poorly understood, which maybe contributed by its rarity in prevalence. A PubMed search reviewed limited literature. Bolat et al. [14] presented the first discussion and concluded that preoperative NLR is not useful in predicting survival rates of patients with GCTs. However, his study was limited by the small sample size (53 patients), poor ROC value (0.55) of the NLR cutoff and the lack of discussion on the relationship between NLR and pathological outcomes. Jankovich et al. [15], in his review of 103 patients, commented a higher prevalence of non-metastatic disease in patients with NLR $<4$. A NLR cutoff of 4 was arbitrarily determined to stay consistent to Templeton et al.'s meta-analysis [21], but this may not represent the optimal cutoff specific to TC. Also, the analyses were univariate, which may not have taken into consideration other pathologic features such as tumour markers, $\mathrm{T}$ staging and tumour size which would have affected the metastatic potential.

Our analysis expands on the previous studies in a larger cohort of patients, with an extended follow-up time and more detailed clinicopathological features to evaluate the association between NLR and TC. We have demonstrated that a higher preoperative NLR $(\geq 3.0)$ predicts LNI and

Table 5. Factors affecting cancer specific survival

\begin{tabular}{lccr}
\hline & Univariate & Multivariate & HR $(95 \% \mathrm{Cl})$ \\
\hline Size $(>4 \mathrm{~cm})$ & 0.851 & 0.758 & \\
Histology & 0.017 & 0.263 & \\
AJCC staging & $<0.01$ & $<0.01$ & $12.37(4.83-19.24)$ \\
NLR & $<0.01$ & 0.042 & $5.11(1.68-11.42)$ \\
\hline
\end{tabular}

$\mathrm{HR}$, hazard ratio; $\mathrm{Cl}$, confidence interval; AJCC, American Joint Committee on Cancer; NLR, neutrophil-to-lymphocyte ratio.

A

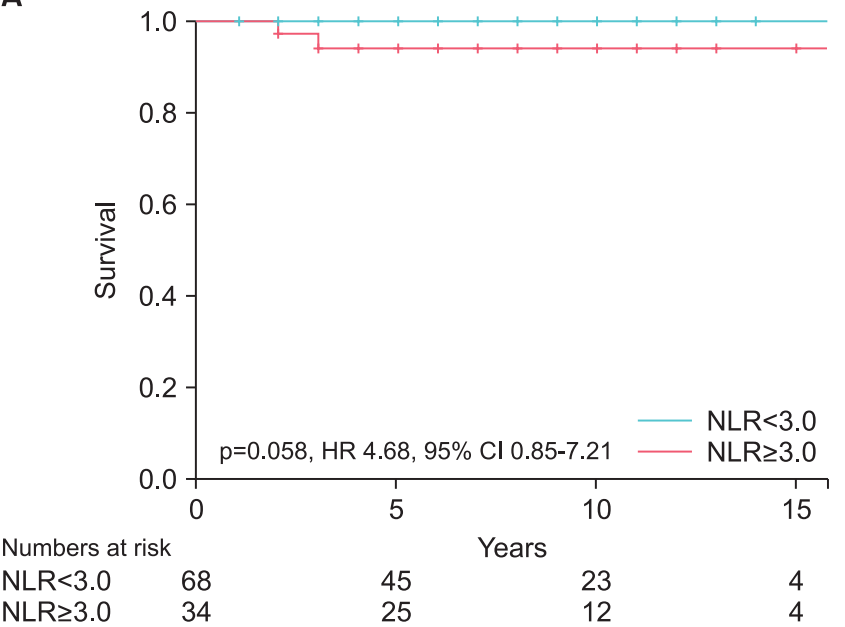

metastatic disease, which hence represent an advanced cancer staging in both seminomatous and NSGCT. There was no evident association between NLR and T staging, and this may reflect the utility of NLR in more advanced disease with metastatic deposits, which is more likely to evoke a systemic response as compared to localized disease.

When considering the association between NLR and survival outcomes, we have demonstrated that NLR mirrored the pathological stage in determining CSS. Patients with NLR $<3.0$ have a 5-year CSS close to $100 \%$, but drops to $\sim 92 \%$ and $76 \%$ in seminomatous and NSGCTs respectively with NLR $\geq 3.0$. As such, NLR could be a useful adjunct in disease prognostication when cancer staging cannot be accurately determined. For instance, in patients with indeterminate LNI or distant metastasis, barring from a histological confirmation with biopsy, concomitant elevated NLR may support a more aggressive treatment. In these otherwise healthy age group patients, clinicians may opt to err on cautious and discuss adjuvant chemotherapy or closer surveillance. While there was no observed association between NLR and recurrence rates, this may be in part attributed to the small recurrence rate and the effects of adjuvant chemotherapy on the blood counts.

We recognize that the study is limited by its retrospective and non-randomized nature. However, prospective study may be limited for the rarity of TC. While the prognosis for TC remains favourable compared to other cancers, it is paramount to appreciate that TC occurs in an otherwise healthy age groups with minimal comorbidities, and our study attempts to streamline additional biomarkers that can complement the TNM staging and tumour markers in prognosticating TC, through identifying a selected

B

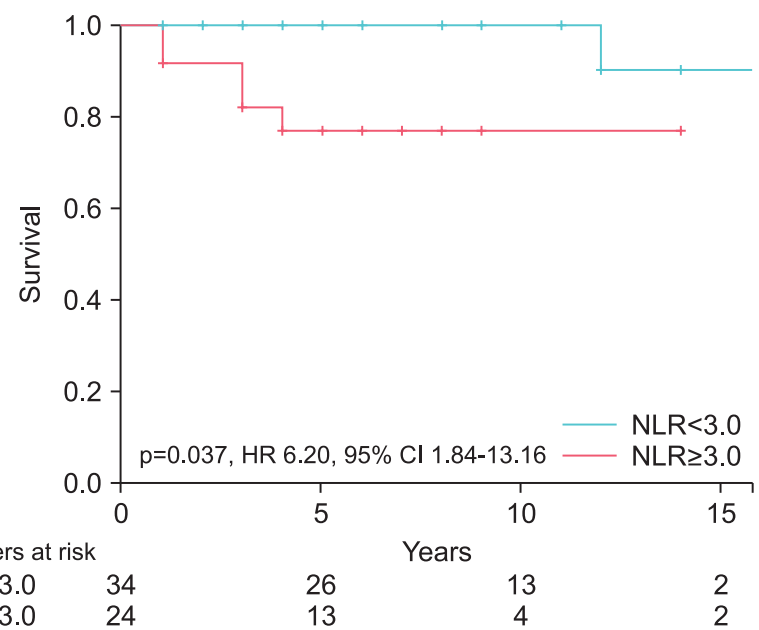

Fig. 3. (A) Neutrophil-to-lymphocyte ratio (NLR) and cancer specific survival (CSS) in seminoma. (B) NLR and CSS in non-seminomatous germ cell tumour (NSGCT). HR, hazard ratio; Cl, confidence interval. 
group of patients with more aggressive tumour biology, as reflected by the NLR. This group of patients may warrant a closer surveillance or consideration of short course adjuvant chemotherapy when distant metastasis remains indeterminate. Our study population is the largest till date in literature with a longer follow-up period. Finally, we recognize that our results are derived from a single, tertiary institution and further external validation will be required.

\section{CONCLUSIONS}

In summary, we recognized that elevated preoperative NLR is independently associated with worse clinicopathologic features, higher cancer staging, high recurrence rate and poorer 5-year CSS. These results suggest that NLR could possibly be a reliable adjunct marker to complement the current TNM staging in patient risk stratification and predicting survival outcomes for patients with TC.

\section{CONFLICTS OF INTEREST}

The authors have nothing to disclose.

\section{REFERENCES}

1. Ferlay J, Steliarova-Foucher E, Lortet-Tieulent J, Rosso S, Coebergh JW, Comber H, et al. Cancer incidence and mortality patterns in Europe: estimates for 40 countries in 2012. Eur J Cancer 2013;49:1374-403.

2. National Cancer Institute, Surveillance, Epidemiology, and End Results Program. Cancer stat facts: testicular cancer [Internet]. National Institutes of Health [updated 2017 Nov; cited 2018 May 18]. Available from: https://seer.cancer.gov/statfacts/html/ testis.html.

3. Znaor A, Lortet-Tieulent J, Jemal A, Bray F. International variations and trends in testicular cancer incidence and mortality. Eur Urol 2014;65:1095-106.

4. Cancer Research UK. Testicular cancer statistics [Internet]. Cancer Research UK [updated 2018 Feb; cited 2018 May 18]. Available from: http://www.cancerresearchuk.org/health-professional/cancer-statistics/statistics-by-cancer-type/testicularcancer.

5. Mantovani A, Allavena P, Sica A, Balkwill F. Cancer-related inflammation. Nature 2008;454:436-44.

6. Zahorec R. Ratio of neutrophil to lymphocyte counts--rapid and simple parameter of systemic inflammation and stress in critically ill. Bratisl Lek Listy 2001;102:5-14.

7. Guthrie GJ, Charles KA, Roxburgh CS, Horgan PG, McMillan DC, Clarke SJ. The systemic inflammation-based neutrophil- lymphocyte ratio: experience in patients with cancer. Crit Rev Oncol Hematol 2013;88:218-30.

8. Jung MR, Park YK, Jeong O, Seon JW, Ryu SY, Kim DY, et al. Elevated preoperative neutrophil to lymphocyte ratio predicts poor survival following resection in late stage gastric cancer. J Surg Oncol 2011;104:504-10.

9. Gomez D, Farid S, Malik HZ, Young AL, Toogood GJ, Lodge JP, et al. Preoperative neutrophil-to-lymphocyte ratio as a prognostic predictor after curative resection for hepatocellular carcinoma. World J Surg 2008;32:1757-62.

10. Ohno Y, Nakashima J, Ohori M, Hatano T, Tachibana M. Pretreatment neutrophil-to-lymphocyte ratio as an independent predictor of recurrence in patients with nonmetastatic renal cell carcinoma. J Urol 2010;184:873-8.

11. Jang WS, Cho KS, Kim KH, Yoon CY, Kang YJ, Lee JY, et al. Prognostic impact of preoperative neutrophil-to-lymphocyte ratio after radical prostatectomy in localized prostate cancer. Prostate Cancer Prostatic Dis 2016;19:298-304.

12. Viers BR, Boorjian SA, Frank I, Tarrell RF, Thapa P, Karnes RJ, et al. Pretreatment neutrophil-to-lymphocyte ratio is associated with advanced pathologic tumor stage and increased cancer-specific mortality among patients with urothelial carcinoma of the bladder undergoing radical cystectomy. Eur Urol 2014;66:1157-64.

13. Tan YG, Eu EWC, Huang HH, Lau WKO. High neutrophilto-lymphocyte ratio predicts worse overall survival in patients with advanced/metastatic urothelial bladder cancer. Int J Urol 2018;25:232-8

14. Bolat D, Aydoğdu Ö, Polat S, Yarımoğlu S, Bozkurt İH, Yonguç T, et al. Predictive value of preoperative neutrophil-to-lymphocyte ratio on the prognosis of germ cell testicular tumors. Turk J Urol 2017;43:55-61.

15. Jankovich M, Jankovichova T, Ondrus D, Breza J. Neutrophilto-lymphocyte ratio as a predictor of preoperative tumor staging in testicular germ cell tumors. Bratisl Lek Listy 2017;118:510-2.

16. Aggarwal BB, Vijayalekshmi RV, Sung B. Targeting inflammatory pathways for prevention and therapy of cancer: shortterm friend, long-term foe. Clin Cancer Res 2009;15:425-30.

17. Schepisi G, Santoni M, Massari F, Gurioli G, Salvi S, Conteduca V, et al. Urothelial cancer: inflammatory mediators and implications for immunotherapy. BioDrugs 2016;30:263-73.

18. Cho H, Hur HW, Kim SW, Kim SH, Kim JH, Kim YT, et al. Pre-treatment neutrophil to lymphocyte ratio is elevated in epithelial ovarian cancer and predicts survival after treatment. Cancer Immunol Immunother 2009;58:15-23.

19. Kumari N, Dwarakanath BS, Das A, Bhatt AN. Role of interleukin-6 in cancer progression and therapeutic resistance. Tumour Biol 2016;37:11553-72. 
20. Müller I, Munder M, Kropf P, Hänsch GM. Polymorphonuclear neutrophils and T lymphocytes: strange bedfellows or brothers in arms? Trends Immunol 2009;30:522-30.

21. Templeton AJ, McNamara MG, Šeruga B, Vera-Badillo FE,
Aneja P, Ocaña A, et al. Prognostic role of neutrophil-to-lymphocyte ratio in solid tumors: a systematic review and metaanalysis. J Natl Cancer Inst 2014;106:dju124. 